\title{
FUZZY DECISION ON OPTIMAL COLLISION AVOIDANCE MEASURES FOR SHIPS IN VESSEL TRAFFIC SERVICE
}

Chien-Min Su

Department of Electrical Engineering, National Taiwan Ocean University, Keelung, Taiwan, R.O.C

Ki-Yin Chang

Department of Merchant Marine, National Taiwan Ocean University, Keelung, Taiwan, R.O.C, b0170@mail.ntou.edu.tw

Chih-Yung Cheng

Department of Electrical Engineering, National Taiwan Ocean University, Keelung, Taiwan, R.O.C

Follow this and additional works at: https://jmstt.ntou.edu.tw/journal

Part of the Transportation Engineering Commons

\section{Recommended Citation}

Su, Chien-Min; Chang, Ki-Yin; and Cheng, Chih-Yung (2012) "FUZZY DECISION ON OPTIMAL COLLISION AVOIDANCE MEASURES FOR SHIPS IN VESSEL TRAFFIC SERVICE," Journal of Marine Science and Technology. Vol. 20: Iss. 1, Article 5.

DOI: $10.51400 / 2709-6998.2420$

Available at: https://jmstt.ntou.edu.tw/journal/vol20/iss1/5

This Research Article is brought to you for free and open access by Journal of Marine Science and Technology. It has been accepted for inclusion in Journal of Marine Science and Technology by an authorized editor of Journal of Marine Science and Technology. 


\title{
FUZZY DECISION ON OPTIMAL COLLISION AVOIDANCE MEASURES FOR SHIPS IN VESSEL TRAFFIC SERVICE
}

\author{
Chien-Min $\mathrm{Su}^{1}$, Ki-Yin Chang ${ }^{2}$, and Chih-Yung Cheng ${ }^{1}$
}

Key words: collision avoidance decision, collision danger domain, fuzzy monitoring system, rudder steering procedure.

\begin{abstract}
This article proposes a new collision avoidance decision method for ships, allowing ships at risk of collision to take timely collision avoidance measures to ensure safe passage. Through vessel traffic service (VTS) collision alert system, an analysis can be made for the probability of a ship collision and an early warning can be issued. For ships in potential collision danger, the proposed method will use Microsoft Visual Studio to establish a knowledge base of international regulations for preventing collisions at sea. Thus, based on an analysis of the situation when encountering other ships, the knowledge base will suggest an appropriate avoidance technique and use a fuzzy monitoring system, propose a novel collision danger domain that forbids entering for give-way ship. Considering the required advance for ship turning, the fuzzy monitoring system will suggest the optimal rudder steering procedure for the give-way ship, allow the ship to avoid collision and ensure navigation safety. The avoidance action taken by the ship does not consider reducing speed, instead focuses only on rudder steering. The system also integrates VTS/automatic identification system (AIS)/marine geographic information system (MGIS) by using MGIS as an AIS imaging system to facilitate the optimal decision for ship collision avoidance and determine the input linguistic variables for the fuzzy logic theory via an analytical hierarchy process. The proposed method can enhance the VTS operator's decision-making abilities for collision avoidance by providing a fuzzy monitoring system.
\end{abstract}

\section{INTRODUCTION}

Many collision incidents are attributed to human error at

Paper submitted 09/22/09; revised 03/16/10; accepted 08/13/10. Author for correspondence: Ki-Yin Chang (e-mail: b0170@mail.ntou.edu.tw).

${ }^{1}$ Department of Electrical Engineering, National Taiwan Ocean University, Keelung, Taiwan, R.O.C.

${ }^{2}$ Department of Merchant Marine, National Taiwan Ocean University, Keelung, Taiwan, R.O.C. sea, particularly during coastal navigation, where ship traffic density is high and marine accidents tend to become more frequent. To achieve the objective of collision avoidance, Collision Avoidance Decision (CAD) research has become an important subject for maritime field. The results from many researchers on collision avoidance automation and intelligence have always focused on the prevention or reduction of collision occurrences in large ship traffic flows or complicated traffic pattern situations. Many researchers propose a ship domain concept as the basis for navigation collision avoidance. Ship domain is a specific range around a ship that other ships should avoid entering. When the Closest Point of Approach (CPA) value between a ship and other ships is smaller than the maximum value of ship domain, the ship or other ships should take action to avoid collision. Fujii \& Tanaka [4] proposed an elliptic ship domain with a long axis length of eight times the ship length and a short axis length of 3.2 times the ship length when analyzing the traffic flow in a specific fairway or water. Goodwin [5] proposed three non-symmetric fan-shaped ship domains to study the required encounter distance when navigating ships in open sea following International Regulations for Preventing Collisions at Sea (COLREGS). Davis et al. [3] used a computer simulation to model the multi-ship encounter and proposed Arena as a collision avoidance action zone consisting of a circle centered at a specific point, with consideration of each ship domain to determine the collision avoidance timing. Coldwell [2] also proposed a ship domain model in an encounter situation in restricted waters. Zhao et al. [18] offered that ship domain boundaries should not be clearly defined and proposed a fuzzy boundary theory. Pietrzykowski [10] focused on restricted waters and narrow channel and used artificial intelligence to propose a fuzzy ship domain as the safety criteria used in the navigating crew decision-making process. Pietrzykowski \& Uriasz [11] focused on encountering ships in open sea and defined fuzzy ship domains of different shapes and sizes based on various factors as safety criteria.

Since ship maneuvering cannot be easily described by a mathematical model, it is impossible to apply to a real-time decision environment. Therefore, in recent years many researchers have begun to incorporate artificial intelligence into collision avoidance research by using neural networks, fuzzy 
techniques and evolutionary computation to study collision avoidance issues. This creates an opportunity for the use of software based calculation of automatic collision avoidance parameters that is not distinctly based on a pure mathematical model [15]. Smeaton \& Coenen [13] used an expert system and knowledge base to assist in both the decision for collision avoidance and the development of an intelligent navigation system at sea. Hiraga et al. [6] adopted a Fuzzy Neural Network (FNN) approach to solving ship collision avoidance issues, using fuzzy rules to infer static and dynamic danger levels and establish decision-making tables for collision avoidance. Zhu et al. [19] used visibility, ship maneuvering performance and CPA direction as input factors and used artificial neural networks to calculate the ship domain for different ship type and visibility. Hwang [7] combined fuzzy theory and $H_{\infty}$ control theory to design a fuzzy collision avoidance system and a $H_{\infty}$ automatic navigation system. Kao et al. [8] used fuzzy logic method to perform a fuzzy ship domain calculation for the ship guarding ring in VTS. Zhuo \& Tang [20] used an artificial neural network to train fuzzy inference system parameters and proposed an intelligent decision-making support system based on fuzzy logic, which investigates anti-collision decision-making in a multi-ship encounter situation. Tsou et al. [17] used genetic algorithm from artificial intelligence to combine COLREGS and ship collision avoidance safety domain within a model emulating biological evolution, generating suggestions for the shortest collision avoidance route and providing the basis for collision avoidance timing and turning angle, timing of ease rudder and rudder angle of course again. Tam et al. [16] focused on encountering ships at a short distance and comprehensively analyzed and studied the collision avoidance techniques and route planning developments proposed by other researchers.

Ship collision avoidance route planning is an issue of multi-criteria and nonlinear planning, demanding a balance point between navigation safety and economics [14]. In other words, the collision avoidance decision must not only maintain the necessary danger assessment and action to avoid collision but also consider minimizing the amount of deviation from the original route. Since there are many challenges to studying automated ship collision avoidance using mathematical models, largely due to uncertainties in sea state, there is no simple, reliable and matured method to study the decision-making process behind collision avoidance. Contrary to conventional research that constructs a ship domain to investigate collision avoidance decision, this article proposes a Fuzzy Collision Danger Domain (FCDD) concept. To effectively establish a ship collision avoidance decision system, a Fuzzy Monitoring System (FMS) developed for the purpose of navigation safety is proposed to analyze ship collision avoidance decision and provide optimal maneuver advice when a ship is in risk of collision. The FMS integrates VTS/AIS/ MGIS and uses ship guarding ring and danger index values from the Collision Alert System (CAS) [9] of VTS. Ship status, static and sea environment data provided by AIS is combined with time and space dependent management concepts within MGIS. The COLREGS provide the basis for collision avoidance when encountering other ships, allowing individual ship encounter situations to be programmed into a Collision Avoidance Knowledge Base (CAKB) using MS Visual Studio 2005. Furthermore, CAS information is used to establish rudder steering procedures with FMS being applied to VTS, allowing give-way ships to take timely and appropriate actions to avoid collision. FMS employs Fuzzy Logic Theory (FLT) to produce a FCDD and prevent other ships from entering. If a ship enters the FCDD, there exists a risk of collision and an appropriate avoidance action and timing is required. This action varies with different encounter situations involving different ship types. This article does not consider slacking speed to avoid collision and instead focuses on ship collision avoidance decision using early rudder steering to achieve the optimal collision avoidance procedures.

The rests of the article are arranged as follows: Section II will discuss the establishment of CAKB to distinguish ship encounter situations and define collision avoidance rules. Section III introduces FMS planning, AIS/MGIS integration and the FCDD concept. Section IV covers the verification of the ship collision avoidance decision mechanism using the MGIS platform to simulate various ship encounter situations and produce the optimal collision avoidance strategy. The conclusion and suggestion for further study are in Section V.

\section{COLLISION AVOIDANCE KNOWLEDGE BASE SYSTEM}

According to COLREGS, there are numerous ship encounter situations, each one varying individual ship responsibilities for collision avoidance action. Part B (steering and asiling rules) section II (conduct of vessels in sight of one another) of COLREGS 72 specifies three ship encounter situations: head-on, crossing and overtaking. However, in reality, inconsistent collision avoidance action occurs frequently among ships where each has a different cognition of the encounter situation. In collision avoidance decision of ships, a clear distinction of the encounter situation is critical.

This research was written in Microsoft Visual Studio 2005 on encountering situation distinction and COLREGS to establish a CAKB as the basis for ship collision avoidance decision. The process diagram of CAKB is shown in Fig. 1.

The ship encounter situation can be divided as follows for CAKB establishment.

An overtaking situation is defined, except when the two ships in sight of one another, as one ship is abaft the other ship abeam in an angle large than $22.5^{\circ}$ (see Fig. 2). At night, only sternlight is visible, not the sidelight and any ship overtaking any other shall keep out of the way of the ship being overtaken.

A head-on situation is defined as two ships are meeting on reciprocal or nearly reciprocal courses so as to involve risk of collision and only exists when two ships see each other. In this 


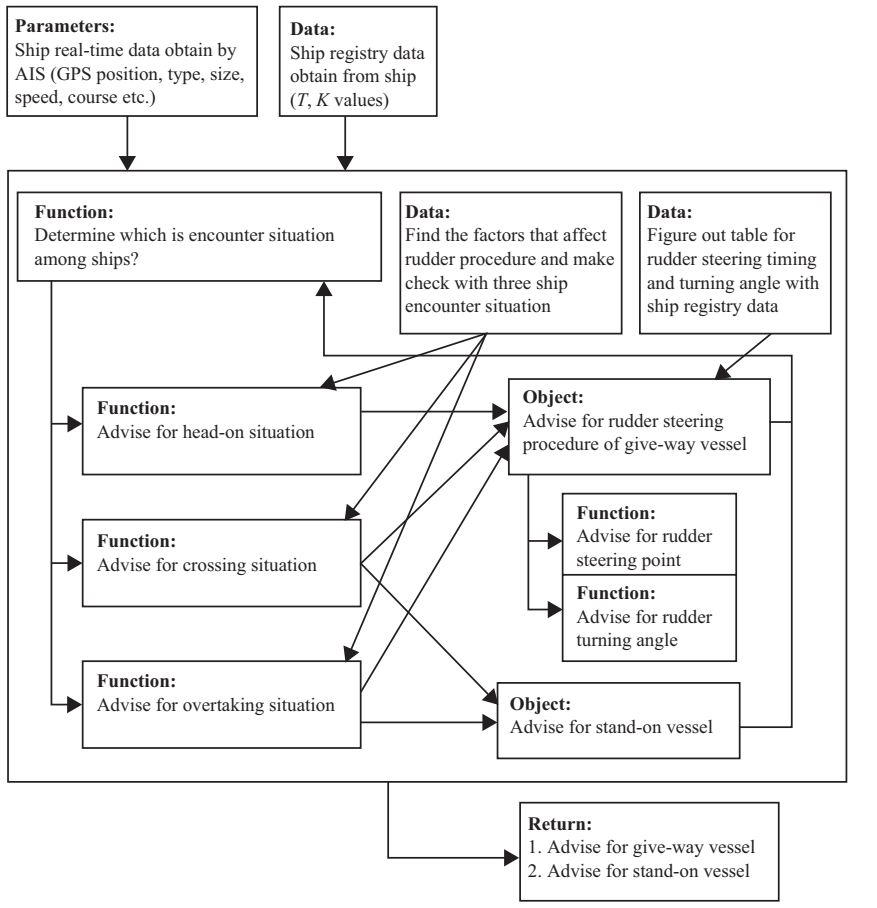

Fig. 1. Process diagram of CAKB.

situation, no yielding relationship exists between the two ships. Regulations require each ship shall alter course to starboard so that each ship shall pass on the port side of the other.

A crossing situation is another encounter that may pose danger besides overtaking and head-on. Regulations require a ship to yield to the other ship on the starboard side and avoid crossing the front of the other ship. Depending on encounter situation, the rights and the responsibilities of the two ships differ, emphasizing the need for clarification on the situation to determine the rights and the responsibilities of each ship and to establish a foundation for collision avoidance decision.

In accordance with the COLREGS, this study focuses on the encounter situations of two ships and divides action modes into six regions labeled $\mathrm{A}$ to $\mathrm{F}$, as shown in Fig. 2. Assuming a heading for ship 1 of $000^{\circ}$, ship 1 is a give-way ship relative to any crossing ship in region $\mathrm{A}\left(005^{\circ} \sim 067.5^{\circ}\right)$ and shall alter course to starboard and avoid collision. Ship 1 is a stand-on ship relative to any crossing ship in region $\mathrm{E}\left(247.5^{\circ} \sim 355^{\circ}\right)$ and is usually not required to take any action to avoid collision. If ship 1 is in an overtaking situation being passed by any ship from the $\mathrm{C}\left(112.5^{\circ} \sim 210^{\circ}\right)$ or $\mathrm{D}\left(210^{\circ} \sim 247.5^{\circ}\right)$ regions, it is usually required to keep course and speed. Ship 1 is a give-way ship relative to any ship from region $\mathrm{B}\left(067.5^{\circ} \sim 112.5^{\circ}\right)$ and is usually required to take action to avoid collision. A head-on situation is created when ship 1 encounters another ship in region $\mathrm{F}\left(005^{\circ} \sim 355^{\circ}\right)$ and in this situation both ships shall alter course to starboard so that each ship shall pass on the port side of the other.

According to ship encounter situations and COLREGS defining stand-on and give-way ships and deciding the rights and

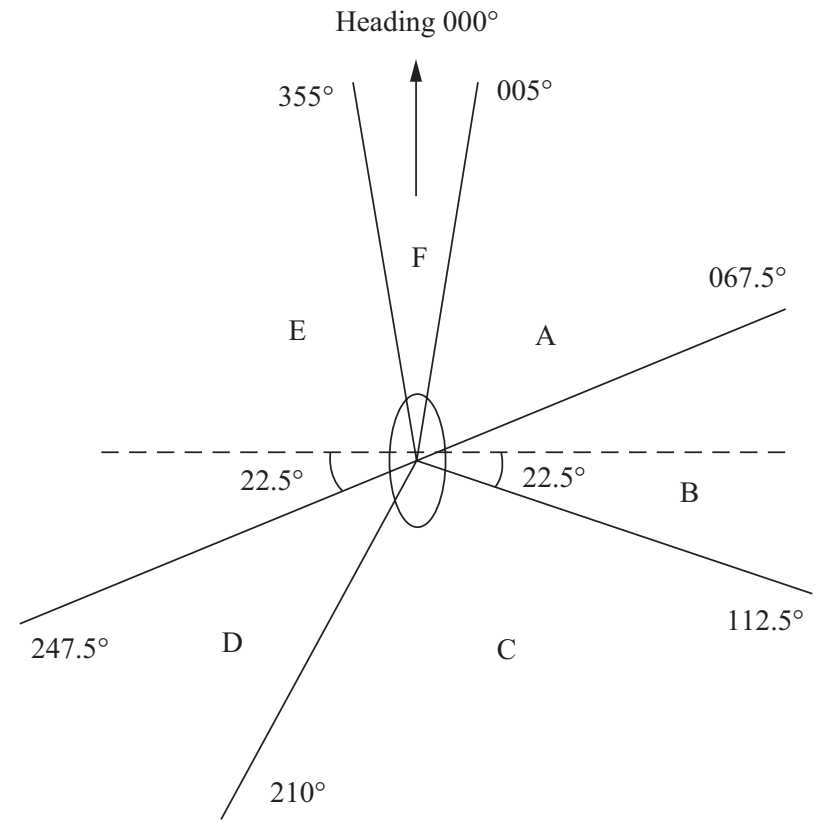

Fig. 2. Diagram of two-ship encounter situation.

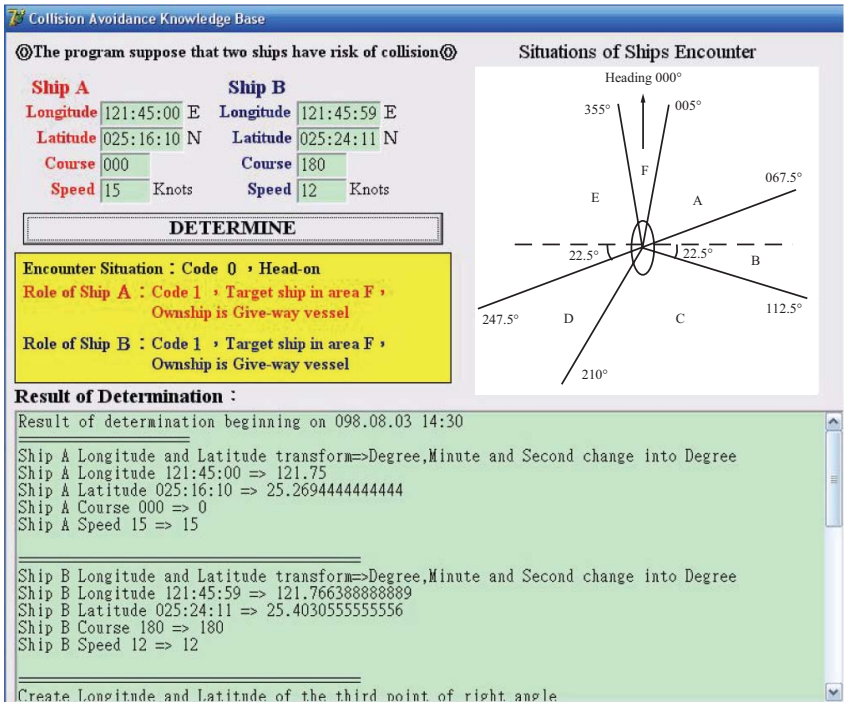

Fig. 3. CAKB system.

responsibilities to form the basis for any collision avoidance action, the CAKB system is proposed as shown in Fig. 3. Navigation information for encountered ships is received by AIS, which is transcoded and instantly written into the database. Using MGIS data access and map presentation modules, dynamic ship information is displayed graphically. Once AIS receives the ship's dynamic and static information, including longitude, latitude, course, speed etc., the CAKB performs an analysis of the situation. This assumes the encountered ship is a target and calculates its relative orientation to determine a location area, and hence defines the encounter situation and the relative rights and responsibilities. The goal is to deter- 
mine the stand-on ship and the give-way ship, allowing both ships to follow COLREGS and take further action to avoid collision.

\section{FUZZY MONITORING SYSTEM}

Ship collision avoidance decision is a very complicated process. It is not only restricted by COLREGS, but also affected to an extent by sea traffic conditions and the personal psychological, physiological and behavioral factors affecting the officers on watch (OOW) on the two encountering ships. For these reasons, ship collision avoidance decision usually undergoes the following procedures: navigation message assessment, encounter situation judgment, collision danger analysis, collision avoidance action and timing decision, decisions of collision avoidance action validity and course again. Any error in this procedure could cause a collision incident. This article describes how to assist VTS monitoring personnel after they obtain a navigation safety message and collision danger level by providing the optimal rudder steering advice to reduce the possibility of harbor and ship collision danger when the CAS of VTS indicates a collision danger between ships.

The CAS of VTS considers the radical axis between the fuzzy guarding rings of two ships and determines a collision danger level based on the radical axis variation. Any collision danger will be reflected on a collision danger index [9]. Due to complicated factors affecting rudder steering timing and turning rudder angle for collision avoidance, an OOW frequently misses the optimal action and timing for collision avoidance when deciding an appropriate action, attributing a large number of collision incidents to human error. In this study, a FMS is developed to provide the optimal collision avoidance rudder steering advice (including rudder steering timing and turning angle) for both the stand-on and give-way ships in an encounter situation using CAKB regulations and assessment results, as well as MGIS analysis and calculation. This process reduces the collision danger for the give-way ship to a safe value and the two ships pass with no contact, eliminating the risk of collision.

Previously researchers focused on the issues surrounding vessel collisions at sea, but few were able to make suggestions to improve the current VTS performance. The Collision Danger Domain (CDD) concept proposed in this article uses FLT to produce FCDD. The FCDD is defined as the safe passing distance for two encountering ships, thus two encountering ships can take collision avoidance actions to avoid entering this domain and ensure navigation safety. The FMS can effectively improve the blind spot found in current VTS monitoring and increase the capability for early collision warning and collision avoidance decision.

\section{System Planning}

To integrate AIS/MGIS, AIS transcoded results are immediately written into the database. Through the MGIS data Access and map presentation modules, dynamic ship

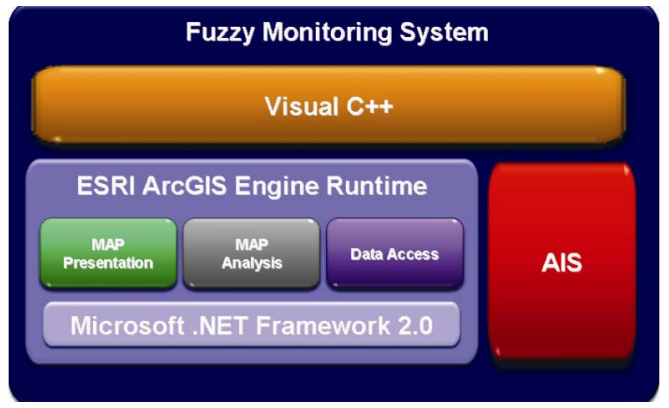

Fig. 4. FMS platform architecture.

information is displayed graphically and verified with the CAKB to determine whether a risk of collision exists. If there is collision danger, colored guarding rings for the two ships are indicated and the give-way and stand-on ships are clearly distinguished. The system then sends the ship information to the FCDD for further analysis. The advance values are used to generate advice on the optimal rudder steering timing and angle for the give-way ship. Different colors are also used to indicate various optimal rudder turning points and rudder angles as well as highlight the optimal rudder steering procedures for collision avoidance.

To perform the complicated information transmission and convert the AIS transcoded results within the database for the CAKB and FCDD, Microsoft Visual Studio 2005 with ESRI ArcGIS Engine Runtime 9.2 was used to develop an AIS transcoding module, a CAKB module and a FCDD module. These were then integrated with the AIS/MGIS platform to produce the complete FMS platform, made up of the architecture shown in Fig. 4. The development of the system used an object-oriented technique and divided the knowledge base modules, including the AIS transcoding, CAKB and FCDD modules, into different classes. These were combined with the independent packages provided by ESRI ArcGIS Engine Runtime 9.2 to encourage repeated use of the program, developing the system through mutual utilization and transmission of parameters, whilst also facilitating the future expansion, deletion and maintenance of the knowledge base module.

\section{Integration of AIS and MGIS}

With the increasing use of AIS to obtain information for VTS and ship collision avoidance decision, it is clear that AIS will be the most important information source available in the future, facilitating improvements to the current collision avoidance methods and increasing navigation safety. Since AIS can provide a large quantity of ship information with real-time characteristics, the FMS platform converts the ship information received by AIS, i.e. ship static data and real-time status data, through the AIS/MGIS interface conversion program, into MGIS accessible information. An example of the AIS transcoded results is shown in Fig. 5. Fig. 5(a) shows the original AIS information, which is converted through the proposed AIS/MGIS interface into useful ship information: 


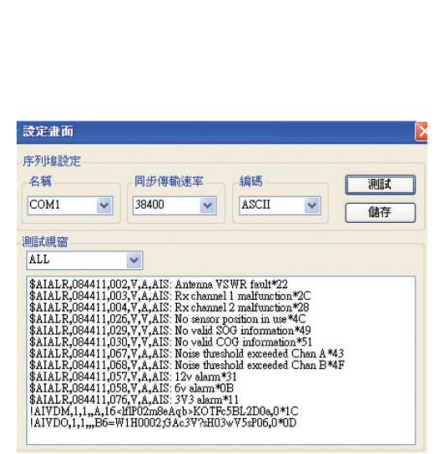

(a) AIS original information

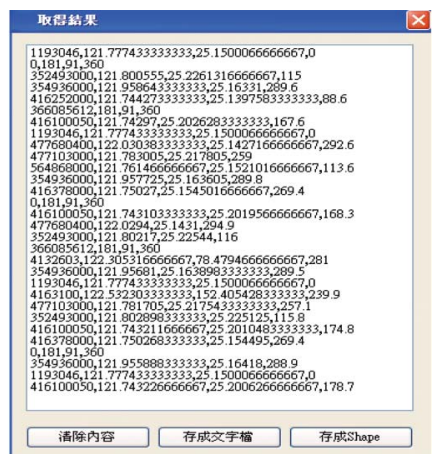

(b) Transcoded ship information
Fig. 5. AIS transcoded results [1].

Maritime Mobile Service Identity (MMSI) code, longitude, latitude, course, and speed etc., as shown in Fig. 5(b). Transcoded ship information can then be stored as a shape file accessible to MGIS, allowing MGIS to be used as a platform to display ship status and achieve the ship monitoring objective.

\section{Fuzzy Collision Danger Domain, FCDD}

This article proposes the use of a FCDD concept whereby an encountering ship is prohibited from entering the FCDD of another ship and collision danger will occur if this rule is breached. In an encounter situation, a ship handled by an OOW will require a different turning degree depending on the relative orientation to the other encountered ship to achieve a safe encounter distance and avoid a collision. Furthermore, any collision avoidance action taken at a given distance from the encountered ship will differ in effect, as the larger the relative bearing of the target ship, the larger the turning angle; and vice-versa. Due to these compounding factors, collision avoidance for ship to ship encounters of varying bearing can prove difficult. Since the minimum safe encountering distance is fuzzy and uncertain, OOWs do not necessarily take collision avoidance action when the minimum safe encounter distance is breached, however they may take collision avoidance action even when the distance between ships is larger than the minimum safe encountering distance. For FMS to suggest a collision avoidance strategy for two encountering ships, FLT is used to generate an FCDD for both ships. The system uses the relative speed of the two ships, ship sizes and sea state as input linguistic variables to produce an output value for FCDD diameter. Using this FCDD, as shown in Fig. 6, FMS can then construct an optimal collision avoidance strategy for the give-way ship. Fig. 6 shows two ships, A and B, in risk of collision. The red arrow represents a 6-minute vector length as the ships are moving, and it is estimated that after 24 minutes the two ships reach the Time of Closest Point of Approach (TCPA), which is indicated by the line $a b$. Since the CPA is too small, the two ships are in risk of collision, prompting the system to use FLT to generate an FCDD based on the encounter situation and compute the distance required

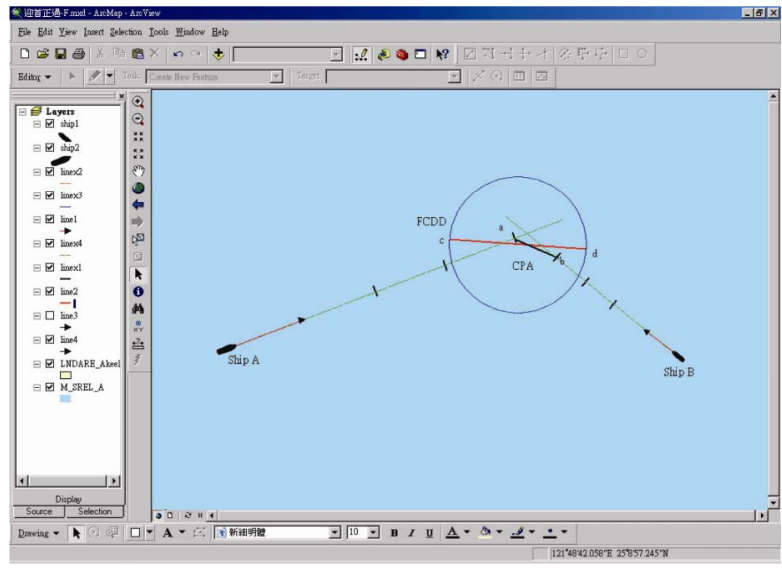

Fig. 6. FCDD map.

for both ships to keep in order to passing by each other safely and preventing a collision. The safe passing distance is the FCDD diameter (i.e. line $c d$ ), hence it can be guaranteed that once the encountering ships take collision avoidance action to keep the ship's passing line tangential to the FCDD without entering the domain, and there will not be a ship collision.

\section{1) Input Linguistic Variables}

When determining an appropriate steering collision avoidance action, many factors can affect the choice of rudder steering timing and rudder angle. These include ship size, speed, sea state, ship type, ship draft, fuel cost, CPA, TCPA, human factor and traffic flow etc. The Analytical Hierarchy Procedure (AHP) [12] has been widely used in both research and practical applications due to its ability to analyze a complicated issue systematically and gain a deeper understanding of the situation, creating a list of prioritized options based on judgments derived from available data. It has the benefits of simplicity, ease of operation and widespread usage among many experts and decision-makers. The choice of input linguistic variables from a range of factors affecting rudder steering timing and rudder angle was made by adopting AHP to establish judgment criteria based on the variables required by the FLT application. The weighting for each factor after AHP solution-seeking is shown in Table 1. From this weighted ranking, ship speed, ship size and sea state were selected as the input linguistic variables.

\section{2) Membership Function}

The degree of membership for FLT input and output linguistic variables can be represented using fuzzy linguistic variables, not only demonstrating the human decision process but also utilizing human empirical rules. Since the system uses FLT to produce a FCDD, Relative ship speed, ship size and sea state were selected as input linguistic variables and FCDD diameter was specified as an output linguistic variable. Using Keelung harbor as a demonstration example, AIS is used to receive the ship dynamic and static information for 100 
Table 1. AHP results.

\begin{tabular}{|c|c|c|}
\hline Factor & Weight & Ranking \\
\hline Ship speed & 0.400 & 1 \\
\hline Ship size & 0.232 & 2 \\
\hline Sea state & 0.113 & 3 \\
\hline Ship type & 0.109 & 4 \\
\hline Ship draft & 0.047 & 5 \\
\hline Traffic flow & 0.045 & 6 \\
\hline Fuel cost & 0.027 & 7 \\
\hline Human factor & 0.026 & 8 \\
\hline
\end{tabular}

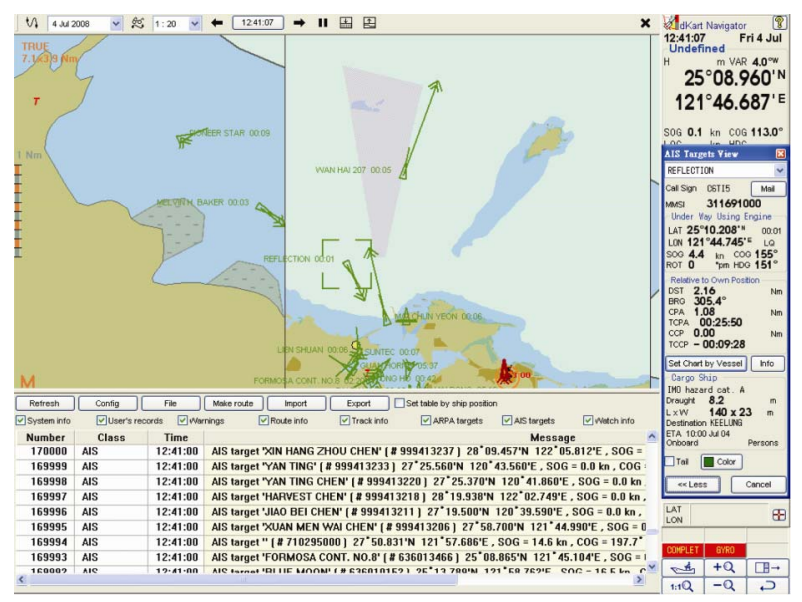

Fig. 7. AIS merchant ship information.

merchant ships (excluding ships anchored, moored or on an inbound or outbound navigation route), as shown in Fig. 7.

The membership functions are defined as follows:

$V$ represents the linguistic variable for relative ship speed. There are three triangular membership functions, including slow, middle and fast. Based on the statistical analysis of AIS collected information from 100 merchant ships, the settings for slow, middle and fast are determined. Since the maximum navigation speed is 22.5 knots and the minimum navigation speed is 3.8 knots, the maximum relative speed is 45 knots and the minimum relative speed is 7.6 knots under all conditions. The average relative speed calculated based on 100 merchant ship speeds is 24.8 knots. Therefore defining "slow" $=[7.6$, 7.6, 24.8], "middle" = [7.6, 24.8, 45] and "fast" = [24.8, 45, 45]. The linguistic set for $V$ is shown in Fig. 8(a) and the membership function variable $x$ is defined as follows:

$$
\begin{aligned}
& \mu_{\text {slow }}(x)=\left\{\begin{array}{ccc}
1 & \text { for } & x \leq 7.6 \\
\frac{24.8-x}{17.2} & \text { for } & 7.6 \leq x \leq 24.8
\end{array}\right. \\
& \mu_{\text {middle }}(x)=\left\{\begin{array}{ccc}
\frac{x-7.6}{17.2} & \text { for } & 7.6 \leq x \leq 24.8 \\
\frac{45-x}{20.2} & \text { for } & 24.8 \leq x \leq 45
\end{array}\right.
\end{aligned}
$$

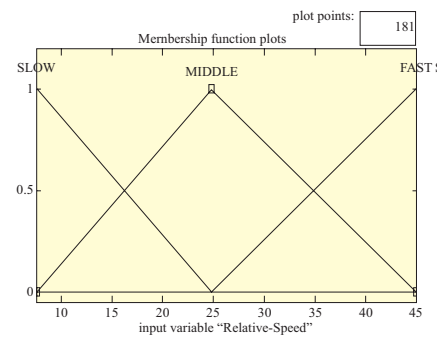

(a) linguistic set for $V$

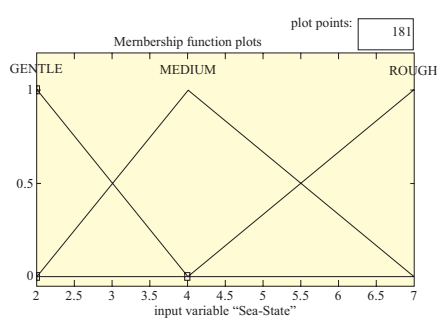

(c) linguistic set for $S$

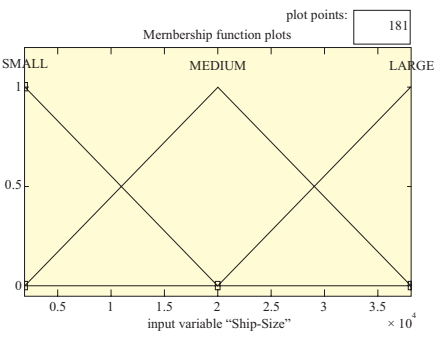

(b) linguistic set for $A$

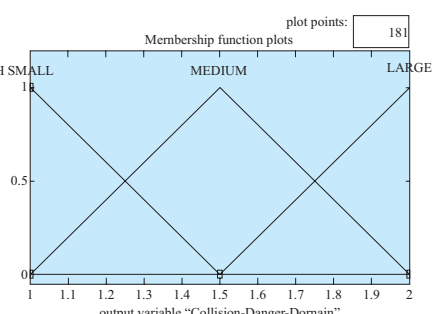

(d) linguistic set for $D$
Fig. 8. Input and output linguistic variable sets.

$$
\mu_{\text {fast }}(x)=\left\{\begin{array}{ccc}
\frac{x-24.8}{20.2} & \text { for } & 24.8 \leq x \leq 45 \\
1 & \text { for } & 45 \leq x
\end{array}\right.
$$

$A$ represents the linguistic variable for ship size. Since the safe distance for encountering ships (FCDD size), ship maneuvering performance and navigation required space are all related to a combination of ship length and width, the ship size parameter is derived by multiplying ship length and ship width. There are three triangular membership functions including small, medium and large. Based on the statistical analysis of AIS collected information from 100 merchant ships, the maximum value of the ship size parameter is $19040 \mathrm{~m}^{2}$ and the minimum value is $960 \mathrm{~m}^{2}$. Thus, the relative maximum encountered ship size is $38080 \mathrm{~m}^{2}$, the minimum value is 1920 $m^{2}$

$$
\begin{gathered}
\mu_{\text {small }}(y)=\left\{\begin{array}{ccc}
1 & \text { for } & y \leq 1920 \\
\frac{20000-y}{18080} & \text { for } & 1920 \leq y \leq 20000
\end{array}\right. \\
\mu_{\text {medium }}(y)=\left\{\begin{array}{ccc}
\frac{y-1920}{18080} & \text { for } & 1920 \leq y \leq 20000 \\
\frac{38080-y}{18080} & \text { for } & 20000 \leq y \leq 38080
\end{array}\right. \\
\mu_{\text {large }}(y)=\left\{\begin{array}{ccc}
\frac{y-20000}{18080} & \text { for } & 20000 \leq y \leq 38080 \\
1 & \text { for } & 38080 \leq y
\end{array}\right.
\end{gathered}
$$

and the average value is $20000 \mathrm{~m}^{2}$. Therefore defining "small" = [1920, 1920, 20000], "medium" = [1920, 20000, 
38080], "large" $=[20000,38080,38080]$. The linguistic set for $A$ is shown in Fig. 8(b) and the membership function variable $y$ is defined as follows:

$S$ represents the linguistic variable for sea state. There are three triangular membership functions including gentle, medium and rough. According to the international sea state force scale, the linguistic variable series for sea state ranges from force 2 to force 7 . An appropriate upper limit is found at a sea state of force 7, which is a high wave situation, under which port jurisdiction will prohibit ships from departure to prevent incidents due to poor weather conditions. The linguistic set for $S$ is shown in Fig. 8(c) and the membership function variable $z$ is defined as follows:

$$
\begin{aligned}
& \mu_{\text {gentle }}(z)=\left\{\begin{array}{ccc}
1 & \text { for } & z \leq 2 \\
\frac{4-z}{2} & \text { for } & 2 \leq z \leq 4
\end{array}\right. \\
& \mu_{\text {medium }}(z)=\left\{\begin{array}{ccc}
\frac{z-2}{2} & \text { for } & 2 \leq z \leq 4 \\
\frac{7-z}{3} & \text { for } & 4 \leq z \leq 7
\end{array}\right. \\
& \mu_{\text {rough }}(z)=\left\{\begin{array}{ccc}
\frac{z-4}{3} & \text { for } & 4 \leq z \leq 7 \\
1 & \text { for } & 7 \leq z
\end{array}\right.
\end{aligned}
$$

$D$ represents the linguistic variable for FCDD diameter. There are three triangular membership functions including small, medium and large. When considering a practical navigation situation, an OOW adopts a collision avoidance measure to keep a safe encounter distance from other ships, taking into account the situation and varying safety distances and avoidance measures accordingly. In general, in the interests of navigation safety, ships are prohibited from passing at too short a distance; while a consideration of economic benefits means the ship encounter distance cannot be too large. Therefore, the range used varies between 1 and 2 nautical miles to keep two ships passing at a safe distance. The linguistic set for $D$ is shown in Fig. 8(d) and the membership function variable $d$ is defined as follows:

$$
\begin{gathered}
\mu_{\text {small }}(d)=\left\{\begin{array}{ccc}
1 & \text { for } & d \leq 1 \\
\frac{1.5-d}{0.5} & \text { for } & 1 \leq d \leq 1.5
\end{array}\right. \\
\mu_{\text {medium }}(d)=\left\{\begin{array}{ccc}
\frac{d-1}{0.5} & \text { for } & 1 \leq d \leq 1.5 \\
\frac{2-d}{0.5} & \text { for } & 1.5 \leq d \leq 2
\end{array}\right.
\end{gathered}
$$

Table 2. Fuzzy inference rules base.

\begin{tabular}{ccccc}
\hline Rule & $V$ & $A$ & $S$ & $D$ \\
\hline$R^{1}$ & slow & small & gentle & small \\
$R^{2}$ & slow & small & medium & medium \\
$R^{3}$ & slow & small & rough & large \\
$\ldots$ & $\ldots$ & $\ldots$ & $\ldots$ & $\ldots$ \\
$R^{25}$ & fast & large & gentle & large \\
$R^{26}$ & fast & large & medium & large \\
$R^{27}$ & fast & large & rough & large \\
\hline
\end{tabular}

( $V$ : Relative speed of ship; $A$ : Ship size; $S:$ Sea state; $D$ : Diameter of CDD)

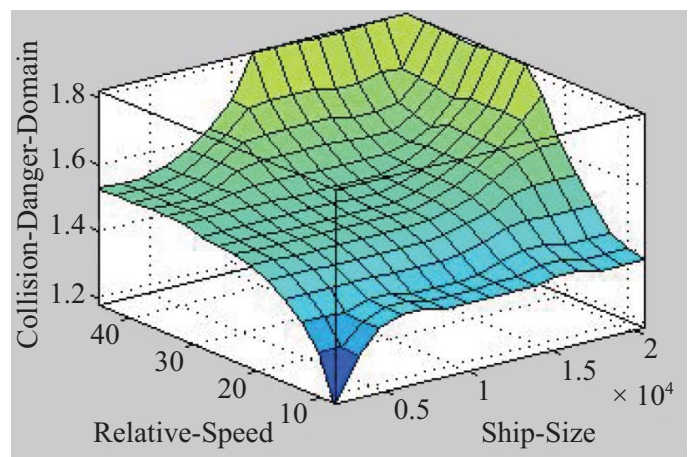

Fig. 9. 3-D diagram for inference rules.

$$
\mu_{\text {large }}(d)=\left\{\begin{array}{ccc}
\frac{d-1.5}{0.5} & \text { for } & 1.5 \leq d \leq 2 \\
1 & \text { for } & 2 \leq d
\end{array}\right.
$$

The generation of an FCDD is an application of a multiinput single-output (MISO) relationship, whose fuzzy inference rule $R$ is generally expressed as:

$R^{i}$ : if $x$ is $A_{i}$ and $y$ is $B_{i}$ then $z$ is $C_{i}, \quad i=1,2, \ldots, n$

where $i$ is the number of fuzzy inference rule.

The FCDD generation includes 27 fuzzy inference rules in total, which have been created based on the simulation of normal intuitive judgment by OOWs. Table 2 shows the fuzzy inference rule base and Fig. 9 shows the 3-D diagram for inference rules.

\section{Rudder Procedure}

After the FCDD is derived, an optimal collision avoidance decision is made based on the factors affecting rudder steering, including ship type and basic maneuvering performance characteristics. Assuming a course line of the give-way ship, various steering rudder angles for collision avoidance are suggested and optimal rudder timing and angle are determined according to the real-time situation, allowing OOWs on the give-way ship to make a decision for collision avoidance to 
Table 3. Basic information for ship model.

\begin{tabular}{|c|c|c|c|c|c|c|c|}
\hline Type & Code & Length $(m)$ & Width $(m)$ & Draft $(m)$ & Course & Speed (kn) & Note \\
\hline Container & ZCONT_50KSF & 290 & 33 & 13 & 000 & 15 & 50,000 tons full load \\
\hline Bulk carrier & ZBULK_90KSF & 272.5 & 32.2 & 12.2 & 180 & 12 & 90,000 tons full load \\
\hline Tanker & ZVLCC_300KSF & 340 & 56 & 22 & 000 & 12 & 300,000 tons full load \\
\hline LNG & ZLNG_137KSF & 297.5 & 45.8 & 11 & 180 & 15 & 137,000 tons full load \\
\hline
\end{tabular}

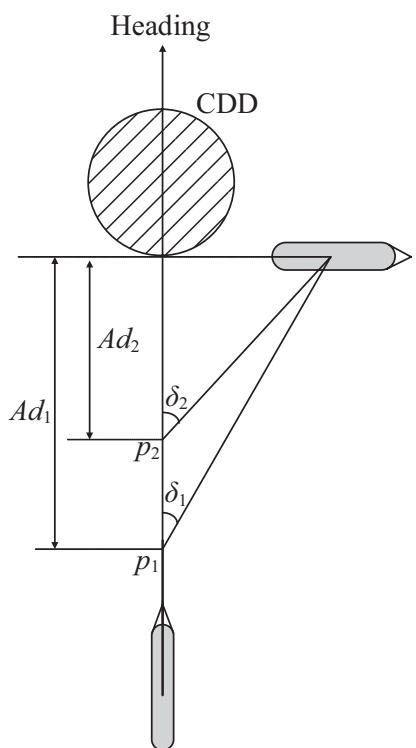

Fig. 10. Relationship between rudder angle and advance.

successfully prevent a collision. A ship moving at a constant linear speed at a constant rudder angle will perform a turning motion. The distance covered from the beginning of the circular movement to the point where the ship's heading turns a certain angle is called advance, or longitudinal distance. Usually, the advance referred to in ship turning data relates to the distance travelled when the heading turns 90 degrees. Without considering the use of deceleration to avoid collision, the system uses the advance as the basis for computing the optimal rudder timing and angle to initiate a turning motion to avoid a collision. However, different ships have various ship maneuverability indices $T, K(T, K$, can be obtained from ship registration information). $T$ and $K$ represent the yaw quick responsibility index and the turning ability index, respectively. Different $T$ and $K$ values will affect ship maneuverability, for instance when $T$ is reduced, the yaw quick responsibility improves. Conversely, when $T$ increases, the yaw quick responsibility worsens. A larger value of $K$ denotes a better turning ability; however a lesser value means the turning ability is reduced.

Fig. 10 describes the required advances for collision avoidance with different turning rudder angles, where $\delta_{1}$ and $\delta_{2}$ represent different turning rudder angles with the relationship $\delta_{1}<\delta_{2}$ and the shaded circular domain represents the CDD. A ship travelling in the original course, initiating a turn at rudder point $p_{1}$ with turning rudder angle $\delta_{1}$ will require an advance $A d_{1}$ to avoid entering the CDD. Conversely, a turn at rudder point $p_{2}$ with turning rudder angle $\delta_{2}$, will require an advance $A d_{2}$. From this, it becomes apparent that $A d_{1}>A d_{2}$. Therefore, when a ship takes a turning collision avoidance method to avoid a CDD and ensure navigation safety, smaller rudder angles require larger advances. When the full rudder $\left(\delta=35^{\circ}\right)$ is used, the required advance is the shortest.

When a ship needs to adopt turning collision avoidance measures, different rudder angles will arise due to differing OOW maneuvering habits and collision avoidance concepts. Regardless of the chosen angle, the proposed FCDD design will need to prevent encountering ships from entering this domain to ensure navigation safety and prevent encountering ships from colliding, while keeping a safe encountering distance. The optimal ship collision avoidance actions should correspond to the original course to determine the optimal rudder timing and angle based on the advance required by the turning rudder. The following approximating equation can be used to calculate the advance $A d$ :

$$
A d=\frac{v}{60} \times t_{90^{\circ}} \times 1852 \times \cos \delta
$$

In Eq. (6), $A d$ : advance $(m)$; $v$ : ship speed begins with the turning $(\mathrm{kn}) ; t_{90^{\circ}}$ : time required for the ship to turn its heading by 90 degrees $(\mathrm{min}) ; \delta$ : turning rudder angle.

\section{SIMULATION RESULTS AND ANALYSIS}

A typical merchant ship without FMS would use a hard port or hard starboard to perform a testing turning circle to figure out the advance, transfer, initial tactical diameter, tactical diameter, lag distance and turning time to assess ship turning degree and the required territorial water space. However, there is no advance data collected for varying rudder angles for the purpose of collision avoidance. The proposed system provides the optimal decision mechanism for ship collision avoidance by using the ship handling simulator SA PILOTSHIP 2000 (As in Fig. 11) from the National Taiwan Ocean University to simulate 4 ships (as in Table 3). Experienced crews were invited to operate the simulator under windfree wave-free sea conditions. The 4 ships were operated with different rudder angles and the testing data was recorded for further analysis.

Based on the course and speed assigned in Table 3, the ship 
Table 4. Advances for the four ships with varying turning rudder angles.

\begin{tabular}{|c|c|c|c|c|}
\hline Rudder advance & $\begin{array}{c}\text { Container } \\
\text { ZCONT_50KSF }\end{array}$ & $\begin{array}{c}\text { Bulk carrier } \\
\text { ZBULK_90KSF }\end{array}$ & $\begin{array}{c}\text { Tanker } \\
\text { ZVLCC_300KSF }\end{array}$ & $\begin{array}{c}\text { LNG } \\
\text { ZLNG_137KSF }\end{array}$ \\
\hline angle & \multicolumn{4}{|c|}{ advance } \\
\hline $\mathrm{R}^{\circ}$ & 1610 & 2030 & 2680 & 2468 \\
\hline $\mathrm{R} 10^{\circ}$ & 1330 & 1604 & 2080 & 2042 \\
\hline $\mathrm{R} 15^{\circ}$ & 1118 & 1306 & 1745 & 1620 \\
\hline $\mathrm{R} 20^{\circ}$ & 1017 & 1088 & 1447 & 1471 \\
\hline $\mathrm{R} 25^{\circ}$ & 950 & 1026 & 1374 & 1321 \\
\hline $\mathrm{R} 30^{\circ}$ & 907 & 939 & 1213 & 1299 \\
\hline R35 & 882 & 862 & 1171 & 1282 \\
\hline
\end{tabular}

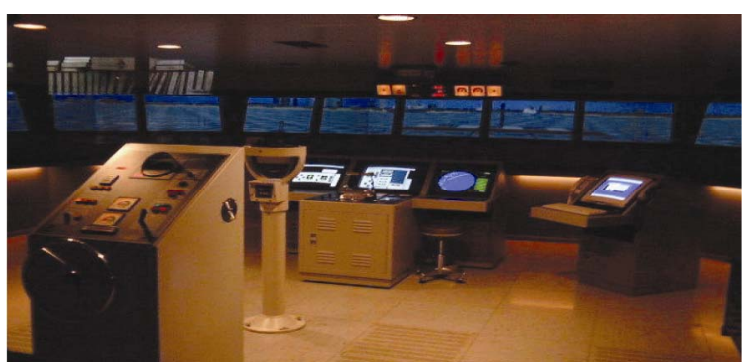

Fig. 11. Ship handling simulator SA PILOTSHIP 2000.

handling simulator was used to model various turning conditions. At the start of turning, the start time, rudder angle and ship position (longitude and latitude) was recorded. At 30 second intervals a recording of position, course, heading and speed was made. When the heading had moved through 90 degrees, the operation was ended and the end time, ship position, course, heading and speed were recorded. The recorded data was saved in an EXCEL spreadsheet and later converted using the geographic information software ArcGIS 9.2 to an MGIS accessible shape file for further analysis and calculation. The advances obtained for the four ships with different turning rudder angles are shown in Table 4.

To validate the test results, different encounter situations including head-on, crossing and overtaking within different environments were simulated using MGIS as a display platform. In each two ship encounter situation with potential collision danger, FMS was started for the encountering ships using CAKB to distinguish the stand-on ship and give-way ship as well as to define rights and responsibilities. FLT is used to derive an FCDD for FMS to make an optimal ship collision avoidance strategy based only on turning collision avoidance, not deceleration collision avoidance. Using the COLREGS 72, the give-way ship shall alter course to starboard for collision avoidance and advance analysis to avoid entering the CDD. The simulation of starboard rudder collision avoidance leads to the optimal rudder point and rudder timing for each rudder angle available to the give-way ship. The following is a case by case analysis of 3 scenarios, detailing the scenario description and simulation results for

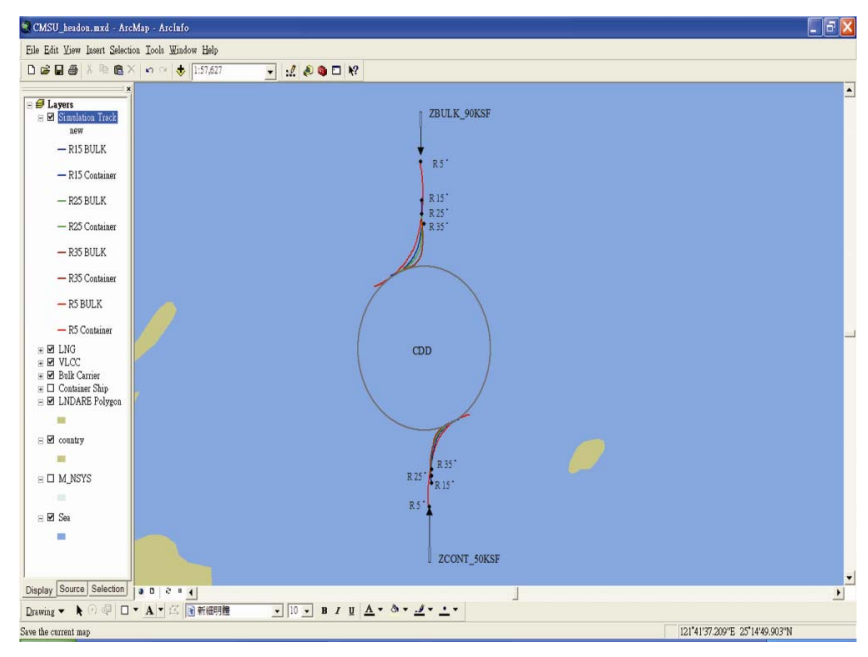

Fig. 12. Optimal collision avoidance decision for two encountering ships (head-on situation).

encountering ships in each case. The simulation sea territory is off the coast of Keelung harbor and sea state is under force 5 . If the rudder point for the expected collision avoidance rudder angle is missed, it means the ship will be unable to avoid entering the CDD and potentially cause collision danger if the particular rudder angle is maintained. This means a larger collision avoidance rudder angle is required to reduce advance to avoid entering the CDD.

\section{CASE 1: head-on}

Two ships, ZCONT_50KSF and ZBULK_90KSF, are in a head-on situation as defined by CAKB. Both ships should alter course to starboard for collision avoidance. The input linguistic variables are: relative ship speed (27 knots), ship size $\left(18344.5 \mathrm{~m}^{2}\right)$ and sea state (force 5). FLT calculates the FCDD diameter to be 1.5 nautical miles. The advance data in Table 4 can be used to derive the rudder point for each turning rudder angle. Fig. 12 shows the two ships implementing the optimal rudder points and rudder steering timing for rudder angles $\mathrm{R} 5^{\circ}, \mathrm{R} 15^{\circ}, \mathrm{R} 25^{\circ}$ and $\mathrm{R} 35^{\circ}$ to avoid a collision. 


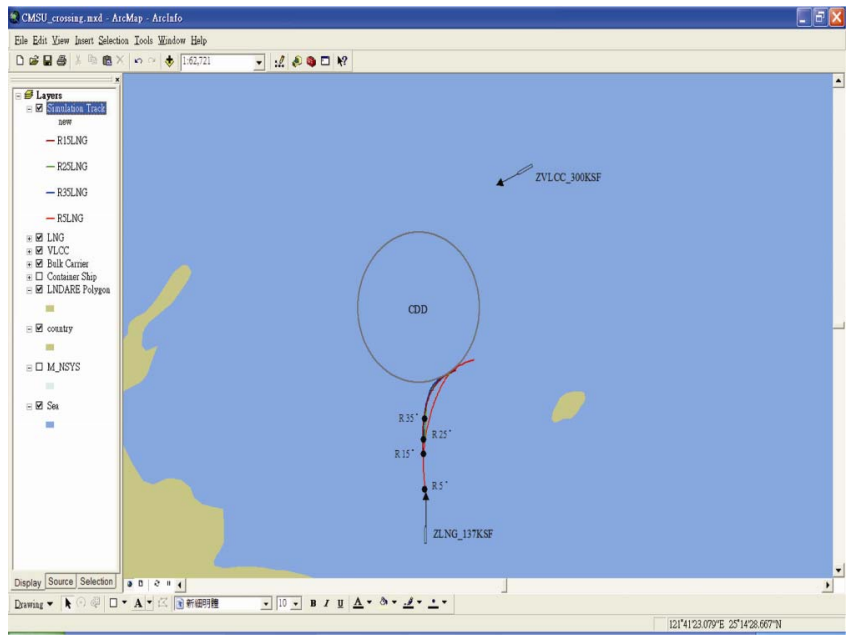

Fig. 13. Optimal collision avoidance decision for two encountering ships (crossing situation).

\section{CASE 2: crossing}

Two ships, ZVLCC_300KSF and ZLNG_137KSF, are in a crossing situation as defined by CAKB. ZVLCC_300KSF sees the starboard side green light of ZLNG_137KSF and takes the role as the stand-on ship, keeping original course and speed. ZLNG_137KSF sees the port side red light of ZVLCC $300 \mathrm{KSF}$ and takes the role as the give-way ship. According to the COLREGS 72, in an encounter situation, the give-way ship should follow left-red right-green regulations and use a starboard rudder to avoid a collision with the stand-on ship. The input linguistic variables are: relative ship speed (27 knots), ship size $\left(32665.5 \mathrm{~m}^{2}\right)$ and sea state (force 5). FLT calculates the FCDD diameter to be 1.62 nautical miles. The advance data in Table 4 can be used to derive the rudder point for each turning rudder angle. Fig. 13 shows the give-way ship ZLNG_137KSF using the optimal rudder points and rudder steering timing for rudder angles $\mathrm{R} 5^{\circ}, \mathrm{R} 15^{\circ}, \mathrm{R} 25^{\circ}$ and $\mathrm{R} 35^{\circ}$ to avoid a collision.

\section{CASE 3: overtaking}

Two ships, ZCONT_50KSF and ZVLCC_300KSF, are in an overtaking situation as defined by CAKB. ZVLCC $300 \mathrm{KSF}$ is the ship being overtaken and is hence the stand-on ship and should keep original course and speed. ZCONT $50 \mathrm{KSF}$ is the overtaking ship and hence the give-way ship, and should adopt starboard or port turning rudder to overtaking. The input linguistic variables are: relative ship speed (27 knots), ship size $\left(28610 \mathrm{~m}^{2}\right)$ and sea state (force 5). FLT calculates the FCDD diameter to be 1.56 nautical miles. The advance data in Table 4 can be used to derive the rudder point for each turning rudder angle. Fig. 14 shows the overtaking ship ZCONT_50KSF overtake the ship ZVLCC_300KSF on the starboard side while employing the optimal rudder points and rudder steering timing for $\mathrm{R} 5^{\circ}, \mathrm{R} 15^{\circ}, \mathrm{R} 25^{\circ}$ and $\mathrm{R} 35^{\circ}$ to avoid a collision.

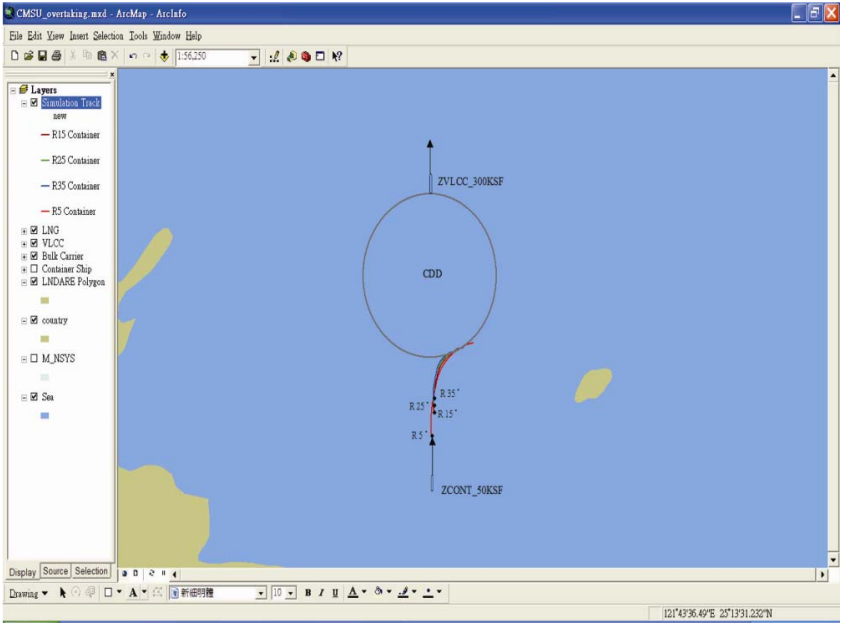

Fig. 14. Optimal collision avoidance decision for two encountering ships (overtaking situation).

\section{CONCLUSIONS}

The new FCDD concept proposed in this article successfully establishes an FMS with the ability to provide an optimal decision for collision avoidance when encountering ships. The previous CAS of VTS established through the integration of VTS/AIS and MGIS can provide a ship guarding ring and danger index. In an encountering situation, based on COLREGS regulations, the CAKB written by Microsoft Visual Studio 2005 will determine the rights and responsibilities for both the encountering ships after clarifying the encounter situation (overtaking, crossing and head-on) and assessing collision avoidance regulations. Once determined, FLT is used to figure out the FCDD, which defines a domain for the encountering ships not to enter while taking action to avoid collision. Finally, since the article does not consider reducing speed, but only altering course to avoid collision, the advance and transfer values required for each rudder turning angle are obtained based on ship handling simulations, allowing FMS to make a collision avoidance decision. To prevent encountering ships from entering the FCDD and causing a collision, FMS will make an early suggestion of the optimal rudder timing and angle to the give-way ship. Future investigation could consider multi-ship encounter situations to develop a more extensive ship collision avoidance decision mechanism.

\section{REFERENCES}

1. Chiu, P. H., Kao, S. L., and Su, C. M., "The integration of AIS and MGIS applied for harbor monitoring," 2008 Conference on Promoting Chinese Seafarer Quality, Taipei, Taiwan, pp. 23-32 (2008). (in Chinese)

2. Coldwell, T. G., "Marine traffic behaviour in restricted waters," The Journal of Navigation, Vol. 36, No. 3, pp. 430-444 (1983).

3. Davis, P. V., Dove, M. J., and Stockel, C. T., "A computer simulation of marine traffic using domains and arenas," The Journal of Navigation, Vol. 33, No. 1, pp. 215-222 (1980).

4. Fujii, Y. and Tanaka, K., "Traffic capacity," The Journal of Navigation, Vol. 24, pp. 543-552 (1971) 
5. Goodwin, E. M., "A statistical study of ship domain," The Journal of Navigation, Vol. 28, No. 3, pp. 328-344 (1975).

6. Hiraga, I., Furuhashi, T., Uchikawa, Y., and Nakayama, S., "An acquisition of operator's rules for collision avoidance using fuzzy neural networks," IEEE Transactions on Fuzzy Systems, Vol. 3, No. 3, pp. 280-287 (1995).

7. Hwang, C. N., "The integrated design of fuzzy collision-avoidance and $H_{\infty}$-autopilots on ships," The Journal of Navigation, Vol. 55, No. 1, pp. 117-136 (2002).

8. Kao, S. L., Lee, K. T., Chang, K. Y., and Ko, M. D., "A fuzzy logic method for collision avoidance in vessel traffic service," The Journal of Navigation, Vol. 60, No. 1, pp. 1-15 (2007).

9. Kao, S. L., Su, C. M., Cheng, C. Y., and Chang, K. Y., "A new method of collision avoidance for vessel traffic service," International Conference on Maritime Technology (ICMT 2007), Taipei, Taiwan, pp. 153-159 (2007).

10. Pietrzykowski, Z., "Ship's fuzzy domain - a criterion for navigational safety in narrow fairways," The Journal of Navigation, Vol. 61, No. 3, pp. 499-514 (2008).

11. Pietrzykowski, Z. and Uriasz, J., "The ship domain - a criterion of navigational safety assessment in an open sea area," The Journal of Navigation, Vol. 62, No. 1, pp. 93-108 (2009).

12. Saaty, T. L., The Analytic Hierarchy Process, McGraw Hill, New York (1980).
13. Smeaton, G. P. and Coenen, F. P., "Developing an intelligent marine navigation system," Computing and Control Engineering Journal, Vol. 1, No. 2, pp. 95-103 (1990).

14. Smierzchalski, R. and Michalewicz, Z., "Modeling of ship trajectory in collision situations by an evolutionary algorithm," IEEE Transactions on Evolutionary Computation, Vol. 4, pp. 227-241 (2000).

15. Statheros, T., Howells, G., and McDonald-Maier, K., "Autonomous ship collision avoidance navigation concepts, technologies and techniques," The Journal of Navigation, Vol. 61, No. 1, pp. 129-142 (2008).

16. Tam, C. K., Bucknall, R., and Greig, A., "Review of collision avoidance and path planning methods for ships in close range encounters," The Journal of Navigation, Vol. 62, No. 3, pp. 455-476 (2009).

17. Tsou, M. C., Kao, S. L., and Su, C. M., "The study of genetic algorithm in ship collision avoidance trajectory planning," 2009 Cross-Straits Conference on Intelligent Transportation Systems, Taichung, Taiwan, pp. 817-832 (2009). (in Chinese)

18. Zhao, J., Wu, Z., and Wang, F., "Comments on ship domains," The Journal of Navigation, Vol. 46, No. 3, pp. 422-436 (1993).

19. Zhu, X., Xu, H., and Lin, J., "Domain and its model based on neural networks," The Journal of Navigation, Vol. 54, No. 1, pp. $97-103$ (2001).

20. Zhuo, Y. and Tang, T., "An intelligent decision system to ship anticollision in multi-ship encounter," Proceedings of the 7th World Congress on Intelligent Control and Automation, Chongqing, China, pp. 1066-1071 (2008). 